\title{
A novel 3RRS wearable fingertip cutaneous device for virtual interaction
}

\author{
Francesco Chinello, Claudio Pacchierotti, \\ Monica Malvezzi, and Domenico Prattichizzo \\ Dept. Information Engineering and Mathematics, University of Siena, Siena, Italy \\ Dept. Advanced Robotics, Istituto Italiano di Technologia, Genova, Italy
}

\section{Introduction}

Wearable haptic devices are recently gaining great popularity among researchers. Such interfaces will indeed enable natural forms of communication between the wearer and the environment. Humans will be free to move and interact with the surrounding world while being provided with rich and unobtrusive haptic information.

In this respect, fingertip cutaneous technologies have shown promising results. Prattichizzo et al. [5] presented a wearable cable-driven 3-degrees-of-freedom (3DoF) cutaneous device for the fingertip. It consists of two parts: one is located on the back of the fingertip, supporting three DC motors, and the other one is placed in contact with the volar surface of the fingertip. The two parts are connected by three cables, actuated by the motors. The mobile platform can press into the user's fingertip and re-angle to simulate contacts with slanted surfaces. Three force sensors placed on the platform measure the contact force at the fingertip. The maximum force applicable is $1.5 \mathrm{~N}$ and the device weights as little as $30 \mathrm{~g}$.

More recently, Tsetserukou et al. [7] presented a 2-DoF wearable fingertip display composed of a five-bar linkage system. Two DC motors, placed on the back of the finger, actuate the linkage mechanism. When the two motors spin in the same direction, the linkage system applies shear stimuli at the finger pulp; when the two motors spin in different directions, the linkage system applies normal stimuli at the finger pulp.

\section{3RRS wearable device}

This paper presents a novel 3RRS wearable fingertip device. It is composed of a static upper body and a mobile end-effector. The upper body ( $\mathrm{F}$ in Fig. 1) is located on the nail side of the finger, supporting three small servo motors $(\mathrm{C})$, and the mobile end-effector $(\mathrm{E})$ is in contact with the finger pulp. The two parts are connected by three articulated legs (A), actuated by the motors. The endeffector can move toward the user's fingertip and rotate it to simulate contacts with arbitrarily-oriented surfaces. A piezoresistive sensor (D) can also measure the force applied by the mobile platform to the fingertip. Finally, a clamp (B) enables the user to easily fasten the device on the finger. Each leg, connecting the endeffector to the upper body, is composed of two rigid links connected to each other, the body, and the end-effector, according to a RRS (Revolute-Revolute-Spherical) kinematic chain. 


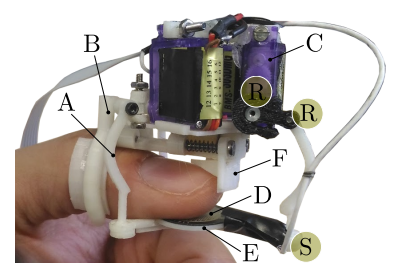

Fig. 1: The 3RRS thimble.

\begin{tabular}{|l|c|}
\hline Controller & Atmega328 \\
\hline Power & 4.8 to $6.0 \mathrm{~V}$ \\
Roll range & $\pm 25^{\circ}$ \\
Pitch range & $\pm 25^{\circ}$ \\
Vertical displacement & $15 \mathrm{~mm}$ \\
Normal force & up to $24 \mathrm{~N}$ \\
\hline
\end{tabular}

Table 1: 3RRS thimble features.

Specifically, three spherical (S) joints connect the distal links of the legs to the end-effector, one revolute $(\mathrm{R})$ joint connects the distal and proximal links of each leg, and another revolute $(\mathrm{R})$ joint connects the proximal link of each leg to the body. The three revolute joints between the proximal links and the body are actuated by the servo motors. In each leg, the axes of the two revolute joints are parallel, so that it constitutes a 2-DoF planar articulated mechanism, constraining the motion of the center of each spherical joint on a plane fixed w.r.t. the body. The mobile end-effector has therefore 3-DoF w.r.t. the body. The proposed device weights $25 \mathrm{~g}$ for $35 \times 50 \times 48 \mathrm{~mm}$ dimensions. A preliminary version was presented in $[1]$.

With respect to the cable-driven fingertip devices presented in $[2,3,4,5,6]$, this device solves the indeterminacy due to the underactuation of the platform. This mechanical structure decouples position and force control problems, simplifying the control structure with respect to the solution proposed in [5].

Acknowledgment: The research has received founding from European Union's Horizon 2020 Research and Innovation Programme, Grant Agreement No. 688857 (SoftPro) and from the European Union Seventh Framework Programme FP7/200\%2013, Grant Agreement No. 601165 (WEARHAP).

\section{References}

1. Chinello, F., Malvezzi, M., Pacchierotti, C., Prattichizzo, D.: Design and development of a 3rrs wearable fingertip cutaneous device. In: Proc. IEEE/ASME International Conference on Advanced Intelligent Mechatronics. pp. 293-298 (2015)

2. Meli, L., Scheggi, S., Pacchierotti, C., Prattichizzo, D.: Wearable haptics and hand tracking via an rgb-d camera for immersive tactile experiences. In: Proc. ACM Special Interest Group on Computer Graphics and Interactive Techniques Conference (2014)

3. Pacchierotti, C., Meli, L., Chinello, F., Malvezzi, M., Prattichizzo, D.: Cutaneous haptic feedback to ensure the stability of robotic teleoperation systems. International Journal of Robotics Research. In Press (2015)

4. Pacchierotti, C., Prattichizzo, D., Kuchenbecker, K.J.: Cutaneous feedback of fingertip deformation and vibration for palpation in robotic surgery. IEEE Transactions on Biomedical Engineering 63, 278-287 (2016)

5. Prattichizzo, D., Chinello, F., Pacchierotti, C., Malvezzi, M.: Towards wearability in fingertip haptics: a 3-dof wearable device for cutaneous force feedback. IEEE Transactions on Haptics 6(4), 506-516 (2013)

6. Prattichizzo, D., Pacchierotti, C., Rosati, G.: Cutaneous force feedback as a sensory subtraction technique in haptics. IEEE Transactions on Haptics 5(4), 289-300 (2012)

7. Tsetserukou, D., Hosokawa, S., Terashima, K.: Linktouch: A wearable haptic device with five-bar linkage mechanism for presentation of two-dof force feedback at the fingerpad. In: Proc. IEEE Haptics Symposium (HAPTICS). pp. 307-312 (2014) 